\title{
Design of a predictive score to assess the risk of developing hypocalcemia after total thyroidectomy. A retrospective study
}

This article was published in the following Dove Press journal: International Journal of General Medicine

\section{Anastasios Papanastasiou (D) \\ Konstantinos Sapalidis' \\ Stylianos Mantalobas' \\ Stefanos Atmatzidis ${ }^{\prime}$ \\ Nikolaos Michalopoulos' \\ Valeriu Surlin (1D) \\ Athanasios Katsaounis' \\ Aikaterini Amaniti (D) \\ Paul Zarogoulidis' \\ loannis Passos' \\ Charilaos Koulouris' \\ Efstathios Pavlidis (D) \\ Dimitrios Giannakidis (D) \\ Stelian Mogoanta ${ }^{2}$ \\ Christoforos Kosmidis (D) \\ Isaak Kesisoglou'}

'3rd Department of Surgery, "AHEPA" University Hospital, Aristotle University of Thessaloniki, Medical School,

Thessaloniki, Greece; ${ }^{2}$ Department of Surgery, Faculty of Dentistry, University of Medicine and Pharmacy of Craiova,

Craiova, Romania
Correspondence: Paul Zarogoulidis 3rd Department of Surgery, "AHEPA" University Hospital, Aristotle University of Thessaloniki, Medical School,

Thessaloniki 5700I, Greece

Tel +30697727 I974

Email pzarog@hotmail.com
Background: Temporary hypocalcemia is the most common complication in patients after total thyroidectomy. To date, according to the literature, various predictors of the above complication have been proposed, but none of them seems to be effective enough.

Objectives: The aim of this study was to develop a reliable predictive tool for biochemical hypocalcemia in the first $48 \mathrm{hrs}$ after total thyroidectomy without central dissection by analyzing several parameters relevant to this operation and to suggest a new score.

Methods: A retrospective study was performed on patients who had undergone total thyroidectomy without central neck dissection from October 2017 until January 2018. Data were collected from 36 patients and studied if there was a statistically significant relationship between the risk of hypocalcemia and 10 preselected prognostic factors.

Results: The prognostic score was formed, which included the 6 factors that showed a statistically significant relationship. Moreover, an extensive check of the predictive value of the above score was performed. It was found, therefore, that at a value of 3 and above the sensitivity was $100 \%$, the specificity $79.16 \%$, the positive prognostic value (PPV) $70.58 \%$ and the negative predictive value (NPV) $100 \%$.

Conclusions: High sensitivity of CaReBe'S TiP score makes it feasible to predict patients with postoperative hypocalcemia. High NPV would allow surgeons to exclude patients with a score less than 3 from supplementary calcium medication and achieve a shorter hospitalization for them.

Keywords: design, thyroidectomy, hypocalcemia, prognostic factors

\section{Introduction}

In Europe, thyroid surgery, regardless of the cause, is ranked third among operations in general surgery. ${ }^{1}$ Total thyroidectomy is recommended according to the guidelines not only for malignant diseases ${ }^{2}$ but for toxic and non-toxic benign multinodular goiter. ${ }^{3}$ Temporary hypoparathyroidism (19-38\%) is by far the most common postoperative complication of total thyroidectomy. ${ }^{4,5}$ Biochemical hypoparathyroidism is the state when the concentration of parathormone $(\mathrm{PTH})$ is under the lower limit according to the microbiological laboratory, accompanied by hypocalcemia. Typically, this limit is $12 \mathrm{pg} / \mathrm{mL} .^{6}$ It is worth noting that hypoparathyroidism, if not treated, always leads to hypocalcemia.

Therefore, it is important to develop a methodology that will be able to identify, in the best and fastest way, an imminent postoperative hypocalcemia. To date, many factors have been associated with the above complication in several studies, with the aim of predicting postoperative hypocalcemia before it even occurs. However, 
none of them is quite effective by itself. ${ }^{5}$ The present study aimed to examine several preoperative and immediate postoperative factors, regarding their possible correlation with developing postoperative biochemical hypocalcemia in the first $48 \mathrm{hrs}$ after total thyroidectomy. Consequently, a prognostic score could be created and the prophylactic medication for hypocalcemia would be prescribed only when it is necessary. The patients, whose calcium concentration would be maintained within normal range according to the predictive score, would be excluded from this treatment.

\section{Patients and methods}

This is a retrospective study and was conducted at the 3rd Surgical Clinic of the University General Hospital of Thessaloniki, "AHEPA", and the study was approved by the investigational review board of the Aristotle University of Thessaloniki. The records of adult patients who had undergone total thyroidectomy from October 2017 until January 2018 were examined after permission by the hospital's ethical committee. Patient written informed consent was acquired for all files-data of this study. All operations were performed by highly experienced surgeons. Inclusion and exclusion criteria were set to define accurately the target population.

Inclusion criteria:

- Patients who undergone total thyroidectomy without central neck dissection regardless of the thyroid disorder.

Exclusion criteria:

- Patients with re-implantation of at least one parathyroid gland (PGRIS score $<4$ ). ${ }^{7}$

- Patients with accidental removal of at least one parathyroid gland found in the histopathologic report (PGRIS score $<4$ ). ${ }^{7}$

- Patients with enlarged central lymph node or papillary thyroid carcinoma $>1 \mathrm{~cm}$ (central neck dissection is performed in such cases).

- Patients with lateral neck dissection.

- Patients with previous thyroid, parathyroid, or other central neck operation.

- No previous medication affecting the calcium serum concentration (calcium supplementation, vitamin D, bisphosphonates, thiazides, denosumamb, bicarbonate, lithium, vitamin A, calcitonin, furosemide, glucocorticoids, estrogens, cinacalcet). ${ }^{8}$
Direct preventive medication is often recommended in these cases because are associated with high prevalence of temporary postoperative hypocalcemia. ${ }^{9}$

A total of 39 total thyroidectomies were performed during the study. However, a total of 36 adult patients were included in the study, since 3 of them met the exclusion criteria. Specifically, two had papillary thyroid carcinoma $>1 \mathrm{~cm}$ and one was treated for osteoporosis that affected calcium concentration. The results of Orloff's et al statement on postoperative hypoparathyroidism and Edafe's et al systematic review and meta-analysis of predictors of post-thyroidectomy hypocalcemia were used after literature review. Ten factors were selected and studied for correlation with temporary hypocalcemia in the first $48 \mathrm{hrs}$, based on our clinical experience and the inclusion/exclusion criteria that were set. ${ }^{5,10}$ These factors are all preoperative, except the PTH which was controlled postoperatively (6 hrs after surgery). The factors studied were age, sex, thyroid gland size, multinodular goiter, retrosternal goiter, ultrasound image of calcifications, malignancy $(<1 \mathrm{~cm})$, thyroid function at initial diagnosis, preoperative total serum calcium concentration, and PTH's concentration after surgery. (Table 1)

Biochemical hypocalcemia was defined as a total serum calcium level $<8.0 \mathrm{mg} / \mathrm{dL}$ corrected for albumin concentration, $\mathrm{Ca}$ [corrected $]=[0.8 \times\{$ normal albumin patient albumin $\}+$ serum Calcium]) or ionized serum calcium $<1.1 \mathrm{mmol} / \mathrm{L}$, based on the physiological limits of the hospital's microbiological laboratory. All patients' calcium levels were corrected for albumin concentration. The under study complication was checked for all patients from the day of surgery until the second postoperative day (6, 24 and 48 hrs after surgery).

The patients were classified into those over and under 50 years old. For the size of the thyroid gland, the most recent ultrasound was taken into account in each patient, indicating whether the size of the gland was normal or not (normal dimensions: height $4 \mathrm{~cm}$, width 15-20 mm, thickness $20-39 \mathrm{~mm}),{ }^{11}$ although the real thyroid volume is usually highly underestimated by this way. ${ }^{12}$ In the same way, the data about the presence or not of multinodular and retrosternal goiter, as well as calcifications were collected. As retrosternal was defined a thyroid goiter in which any part of the gland extends below the thoracic inlet with the patient in the surgical position. ${ }^{13}$ Fine needle aspiration (FNA) was performed on patients with a suspicious nodule for malignancy. Based on the result of this test, which is imprinted with the Bethesda scale (1-6), patients were 
Table I Demographic data per factor

\begin{tabular}{|c|c|c|}
\hline Variables & $\begin{array}{l}\text { With post- } \\
\text { operative } \\
\text { hypocalcemia } \\
(n=12)\end{array}$ & $\begin{array}{l}\text { Without post- } \\
\text { operative } \\
\text { hypocalcemia } \\
(n=24)\end{array}$ \\
\hline Age & $\begin{array}{l}>50: 9 \\
\leq 50: 3\end{array}$ & $\begin{array}{l}>50: 19 \\
\leq 50: 5\end{array}$ \\
\hline Sex & $\begin{array}{l}\text { Male: I } \\
\text { Female: II }\end{array}$ & $\begin{array}{l}\text { Male: } 7 \\
\text { Female: } 17\end{array}$ \\
\hline Thyroid gland size & $\begin{array}{l}\text { Large: II } \\
\text { Normal: I }\end{array}$ & $\begin{array}{l}\text { Large: } 12 \\
\text { Normal: } 12\end{array}$ \\
\hline Multinodular goiter & $\begin{array}{l}\text { Yes: } 8 \\
\text { No: } 4\end{array}$ & $\begin{array}{l}\text { Yes: } 15 \\
\text { No: } 9\end{array}$ \\
\hline Retrosternal goiter & $\begin{array}{l}\text { Yes: } 6 \\
\text { No: } 6\end{array}$ & $\begin{array}{l}\text { Yes: } 3 \\
\text { No: } 21\end{array}$ \\
\hline Calcifications & $\begin{array}{l}\text { Yes: } 7 \\
\text { No: } 5\end{array}$ & $\begin{array}{l}\text { Yes: } 10 \\
\text { No: } 14\end{array}$ \\
\hline Malignancy & $\begin{array}{l}\text { Bethesda } \geq 4: 8 \\
\text { Bethesda }<4: 3 \\
\text { and no FNA: I }\end{array}$ & $\begin{array}{l}\text { Bethesda } \geq 4: 6 \\
\text { Bethesda }<4: 12 \text { and } \\
\text { no FNA: } 6\end{array}$ \\
\hline $\begin{array}{l}\text { Thyroid function at } \\
\text { initial diagnosis }\end{array}$ & $\begin{array}{l}\text { Pathologic: } 10 \\
\text { Normal: } 2\end{array}$ & $\begin{array}{l}\text { Pathologic: } 9 \\
\text { Normal: } 15\end{array}$ \\
\hline Preoperative calcium & $\begin{array}{l}<9: 7 \\
\geq 9: 5\end{array}$ & $\begin{array}{l}<9: 3 \\
\geq 9: 21\end{array}$ \\
\hline $\begin{array}{l}\text { PTH } 6 \text { hrs after } \\
\text { surgery }\end{array}$ & $\begin{array}{l}<1.58: 10 \\
\geq 1.58: 2\end{array}$ & $\begin{array}{l}<1.58: 9 \\
\geq 1.58: 15\end{array}$ \\
\hline
\end{tabular}

ranked to those who had the lowest chance of suffering from thyroid malignancy (Bethesda 2-3) and those who were most likely or proven that have malignancy (Bethesda 4-6). It should be noted that patients who did not have an FNA test because there was no relevant clinical or imaging evidence were classified with those in the first category. Furthermore, thyroid function in the initial diagnosis was tested with thyroid stimulating hormone (TSH), classifying patients into euthyroid, hypothyroid and hyperthyroid. The preoperative serum calcium concentration was controlled during the patient's hospital admission the day before surgery. With a limit of $9 \mathrm{mg} / \mathrm{dL}$ preoperative calcium, patients were classified into two subcategories. ${ }^{14}$ Finally, information on whether or not the patients had normal serum levels of PTH was collected 6 hrs after surgery, according to the hospital's microbiology laboratory $(1.58-6.03 \mathrm{pmol} / \mathrm{L})$.

\section{Statistical analysis}

The SPSS 25 software was used. The sample was initially divided into two groups, depending on whether they had biochemical hypocalcemia in the first $48 \mathrm{hrs}$ after the operation. Subsequently, statistical analysis was performed for each individual independent variable to ascertain if there was a statistically significant correlation between them and the under study complication. The above comparisons were performed with the odds ratio (OR) test $(p<0.05)$. Independent variables that displayed a statistically significant relationship to the risk of developing hypocalcemia were then included in the prognostic score. Finally, all patients were ranked according to the score and the sensitivity, the specificity, the PPV and the NPV for each possible value of the score were calculated.

\section{Results}

Out of 36 patients, $12(33.3 \%)$ developed biochemical hypocalcemia in the first $48 \mathrm{hrs}$ after total thyroidectomy. There was no statistically significant correlation between the dependent variable and age $(p=0.777)$, sex $(p=0.184)$, multinodular goiter $(p=0.806)$ and calcifications $(p=0.348)$. On the contrary, all the remaining factors significantly affect the risk of postoperative biochemical hypocalcemia. These factors are the large thyroid gland's size $(p=0.032)$, the ultrasound image of retrosternal goiter $(p=0.021)$, the hypothyroid or hyperthyroid patient's status $(p=0.016)$, the risk of malignancy based on a previous FNA test (Bethesda $\geq 4)(p=0.02)$, the preoperative calcium concentration $<9 \mathrm{mg} / \mathrm{dL}(p=0.007)$ and the concentration of postoperative PTH $<1.58 \mathrm{pmol} / \mathrm{L}(p<0.001)$. (Table 2$)$

According to the above data, a prognostic score was developed, which would allow the early detection of temporary biochemical hypocalcemia after total thyroidectomy without central neck dissection. This included all the independent variables that displayed a statistically significant relationship with biochemical hypocalcemia. Overall, this consists of 6 prognostic factors. Each of them gives 1 point to the score ( $0-6$ points). To achieve an easier memorization of the score, the name CaReBe'S TiP was given, which is an acronym from the initials of the included factors. (Table 3)

Moreover, diagnostic tests were performed for the CaReBe'S TiP score to determine whether this is reliable and useful. Sensitivity, specificity, PPV and NPV were calculated for each possible value of the score. It is worth noting, that for a score value $\geq 3$ the sensitivity is 
Table 2 Results with the use of odds ratio test

\begin{tabular}{|l|l|l|l|}
\hline Independent variables & OR & P-value & $\begin{array}{l}\text { Statistically significant } \\
\text { relationship }\end{array}$ \\
\hline I. Age $>50$ & 1.267 & 0.777 & No \\
2. Sex & 0.221 & 0.184 & No \\
3. Multinodular goiter & 1.2 & 0.806 & No \\
4. Ultrasound image of calcifications & 1.96 & 0.348 & No \\
5. Large thyroid gland's size & 11 & 0.032 & Yes \\
6. Retrosternal goiter & 7 & 0.021 & Yes \\
7. Thyroid function in the initial diagnosis & 8.33 & 0.016 & Yes \\
8. Suspected or proven malignancy based on FNA test & 6 & 0.02 & Yes \\
9. Preoperative Calcium <9.00 mg/dL & 9.8 & 0.007 & Yes \\
10. PTH <I.58 pmol/L 6 hrs postoperatively & 55 & $<0.001$ & Yes \\
\hline
\end{tabular}

Table 3 Predictive score to assess the risk of postoperative hypocalcemia after total thyroidectomy (CaReBe'S TiP)

\begin{tabular}{|c|c|c|c|}
\hline Variables & & Yes & No \\
\hline Total serum calcium <9 mg/dL preoperatively (Calcium) & $\mathrm{Ca}$ & 1 & 0 \\
\hline Ultrasound image of retrosternal goiter (Retrosternal goiter) & $\operatorname{Re}$ & 1 & 0 \\
\hline Bethesda $\geq 4$ based on FNA & $\mathrm{Be}$ & 1 & 0 \\
\hline Large thyroid gland according to ultrasound (Size) & 'S & I & 0 \\
\hline $\begin{array}{l}\text { Hyperthyroid/Hypothyroid patient in the initial } \\
\text { diagnosis (TSH) }\end{array}$ & $\mathrm{Ti}$ & I & 0 \\
\hline PTH $<$ I.58 pmolLI 6 hrs after the operation (PTH) & $\mathrm{P}$ & 1 & 0 \\
\hline Overall & CaReBe'S TiP & 7 & 0 \\
\hline
\end{tabular}

$100 \%$ and the specificity is $79.16 \%$. Respectively, when the score has a value $\geq 4$, the sensitivity is $83.3 \%$ and the specificity is $100 \%$. (Table 4 )

PTH is considered to be the best predictor for the under study complication by many. ${ }^{15}$ For this reason, the predictive indicators of CaReBe'S TiP score were compared with those of PTH alone. CaReBe'S Tip score appears to be a better predictive test. This is also illustrated in the figure below where the respective ROC curves are compared (Figure 1).

\section{Discussion}

It is estimated that the predictive score developed may have a significant prognostic value regarding the risk of postoperative biochemical hypocalcemia following total thyroidectomy without central neck dissection. At a scoring value $\geq 3$, the sensitivity is $100 \%$, so it has detected all the patients, who had the complication. At the same time, the NPV is $100 \%$ and the PPV of $70.58 \%$, which means that patients with scoring values ranging from 0 to 2 did not suffer from biochemical hypocalcemia, which was $70.58 \%$ likely to occur in patients with a scoring value from 3 to 6 . However, the specificity in the above scoring value is $79.16 \%$, so there were also detected $20.84 \%$ patients without hypocalcemia, who essentially had a false-positive result. Respectively, at a value $\geq 4$, the sensitivity is $83.3 \%$ and the specificity $100 \%$. The high specificity means that a positive result in the score will only identify patients who postoperatively have biochemical hypocalcemia. However, some of them will not be detected because of the lower sensitivity.

Table 4 Evaluation of predictive power of CaReBe'S TiP score

\begin{tabular}{|c|c|c|c|c|c|c|}
\hline Score & $\begin{array}{l}\text { Group A, n=24 (Patients without } \\
\text { hypocalcemia) }\end{array}$ & $\begin{array}{l}\text { Group B, n= I } 2 \\
\text { (Patients with } \\
\text { hypocalcemia) }\end{array}$ & Sens. & Spec. & PPV & NPV \\
\hline$\geq 3$ & 5 & 12 & $100 \%$ & $79.16 \%$ & $70.58 \%$ & $100 \%$ \\
\hline$\geq 4$ & 0 & 10 & $83.3 \%$ & $95.83 \%$ & $90.9 \%$ & $92 \%$ \\
\hline
\end{tabular}



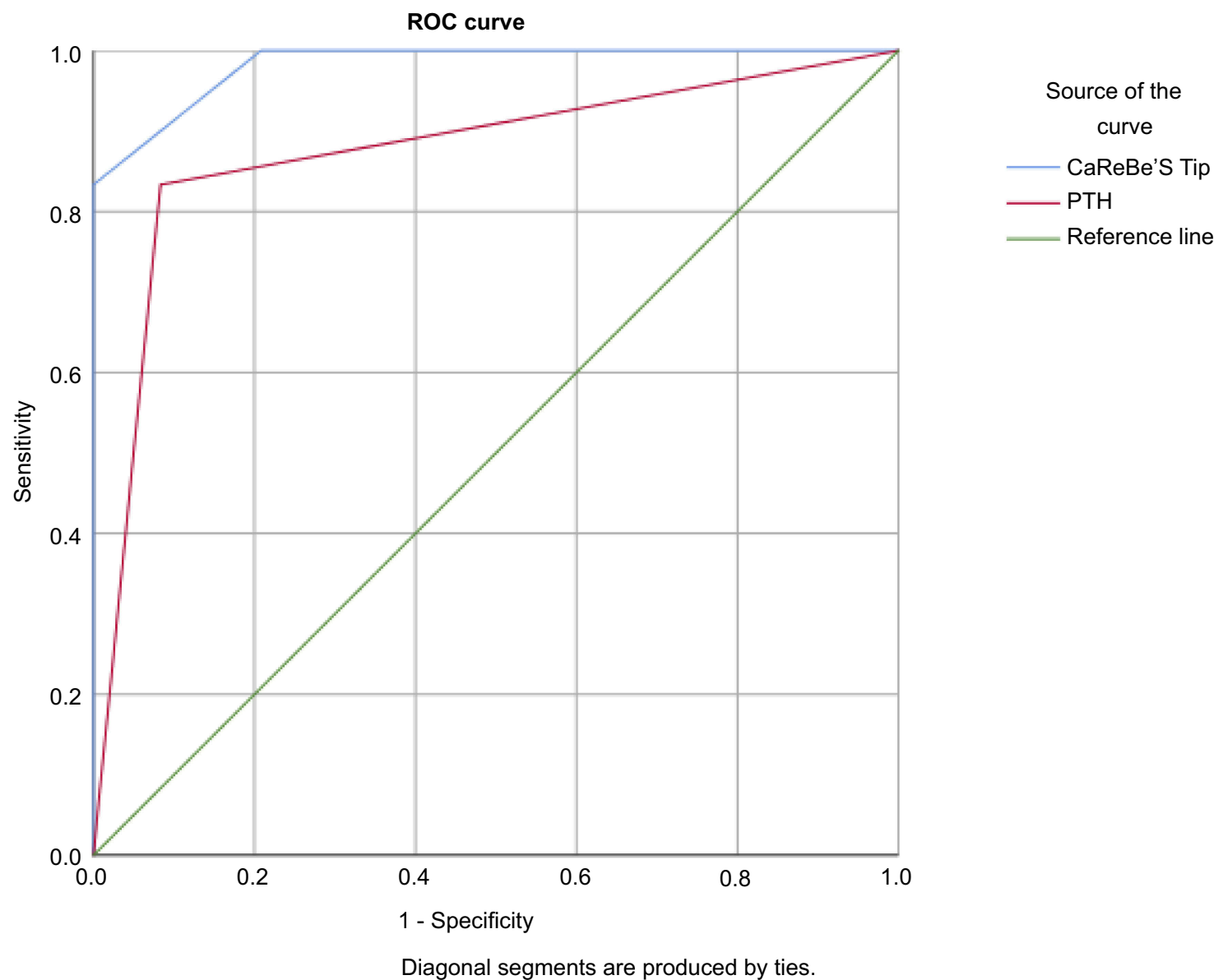

Therefore, it is considered that the more acceptable scoring value is $\geq 3$ as a screening test. In this way, the two main objectives are achieved. First, all patients who would experience postoperative hypocalcemia could be identified. A prophylactic treatment, including oral calcium supplementation with or without calcitriol, should be administered to them as soon as possible. A few falsepositive results are inevitable. The prophylactic medication would also be received from these patients. It should be noted that this treatment has few potential side effects, which are reversed quickly after termination of the medication. ${ }^{16}$ Second, patients with a scoring value $<3$ would not have biochemical hypocalcemia. Therefore, they should be excluded from prophylactic treatment. Consequently, a more targeted therapeutic approach could be achieved.

A further predictive score with 8 prognostic factors for the same purpose in the international bibliography has been published by Ashim Sarkar et al. ${ }^{17}$ The sample of patients is approximately the same, and similar statistical methods are used. One fundamental difference of the above study is that this was prospective that adds more credibility to the data collected. On the other hand, the scoring value with the highest sensitivity is combined with much lower specificity than those demonstrated in the current study. Also, the postoperative calcium $12 \mathrm{hrs}$ after surgery has been chosen as a predictive factor by Sarkar et al, making the prognostic test less timely. Finally, another limitation of the above research is the predictive factor concerning the excised parathyroid glands, since it is indicative of prophylactic treatment for hypocalcemia. ${ }^{9}$

In conclusion, CaReBe'S TiP predictive score could be applied to the clinical practice to check its reliability and validity, given that it is not detrimental to any patient. However, it would be preferable to initiate a new prospective research by studying the same risk factors. In addition, the risk factor surgeon should be always taken seriously into account, which could not be included in the current study. ${ }^{18}$ Also, it is necessary for a larger sample of patients in order to be more representative of the 
population and to develop a multivariable logarithmic regression model. In this way, it is likely to create a much more reliable predictive score that could be used effectively as a screening test. It is therefore essential to carry out further research to obtain more accurate and valid results.

\section{Disclosure}

The authors report no conflicts of interest in this work.

\section{References}

1. Dionigi G. Surgery for benign thyroid disease in 2018. Gland Surg. 2018;7(2):239-241. doi:10.21037/gs.2017.12.07

2. Haugen BR. 2015 American Thyroid Association Management Guidelines for adult patients with thyroid nodules and differentiated thyroid cancer: what is new and what has changed? Cancer. 2017;123 (3):372-381. doi:10.1002/cncr.30360

3. Ross DS, Burch HB, Cooper DS, et al. 2016 American Thyroid Association Guidelines for diagnosis and management of hyperthyroidism and other causes of thyrotoxicosis. Thyroid. 2016;26 (10):1343-1421. doi:10.1089/thy.2016.0229

4. Hauch A, Al-Qurayshi Z, Randolph G, Kandil E. Total thyroidectomy is associated with increased risk of complications for low- and high-volume surgeons. Ann Surg Oncol. 2014;21(12):3844-3852. doi:10.1245/s10434-014-3846-8

5. Edafe O, Antakia R, Laskar N, Uttley L, Balasubramanian SP. Systematic review and meta-analysis of predictors of postthyroidectomy hypocalcaemia. Br J Surg. 2014;101(4):307-320. doi: $10.1002 /$ bjs. 9384

6. Stack BC Jr, Bimston DN, Bodenner DL, et al. American Association of Clinical Endocrinologists and American College of Endocrinology Disease State Clinical Review: postoperative hypoparathyroidismdefinitions and management. Endocr Pract. 2015;21(6):674-685. doi:10.4158/EP14462.DSC
7. Sitges-Serra A. The PGRIS and parathyroid splinting concepts for the analysis and prognosis of protracted hypoparathyroidism. Gland Surg. 2017;6(Suppl 1):S86-S93. doi:10.21037/gs.2017.07.16

8. Ruppe MD. Medications that affect calcium. Endocr Pract. 2011;17 (Suppl 1):26-30. doi:10.4158/EP10281.RA

9. Palazzo FF, Sywak MS, Sidhu SB, Barraclough BH, Delbridge LW. Parathyroid autotransplantation during total thyroidectomy-does the number of glands transplanted affect outcome? World J Surg. 2005;29(5):629-631. doi:10.1007/s00268005-7729-9

10. Orloff LA, Wiseman SM, Bernet VJ, et al. American Thyroid Association Statement on postoperative hypoparathyroidism: diagnosis, prevention, and management in adults. Thyroid. 2018;28 (7):830-841. doi:10.1089/thy.2017.0309

11. Mohebati A, Shaha AR. Anatomy of thyroid and parathyroid glands and neurovascular relations. Clin Anat. 2012;25(1):19-31. doi:10.1002/ca.21220

12. Miccoli P, Minuto MN, Orlandini C, Galleri D, Massi M, Berti P. Ultrasonography estimated thyroid volume: a prospective study about its reliability. Thyroid. 2006;16(1):37-39. doi:10.1089/ thy.2006.16.37

13. Huins CT, Georgalas C, Mehrzad H, Tolley NS. A new classification system for retrosternal goitre based on a systematic review of its complications and management. Int $J$ Surg. 2008;6(1):71-76. doi:10.1016/j.ijsu.2007.02.003

14. Amir A, Sands NB, Tamilia M, Hier MP, Black MJ, Payne RJ. Preoperative serum calcium levels as an indicator of postthyroidectomy hypocalcemia. J Otolaryngol Head Neck Surg. 2010;39 (6):654-658.

15. Graff AT, Miller FR, Roehm CE, Prihoda TJ. Predicting hypocalcemia after total thyroidectomy: parathyroid hormone level vs. serial calcium levels. Ear Nose Throat J. 2010;89(9):462-465.

16. Roh JL, Park JY, Park CI. Prevention of postoperative hypocalcemia with routine oral calcium and vitamin D supplements in patients with differentiated papillary thyroid carcinoma undergoing total thyroidectomy plus central neck dissection. Cancer. 2009;115 (2):251-258. doi: 10.1002/cncr.24027

17. Sarkar AJS, Basak B, Gayen GC, Sit S, Sarkar A. Evaluation of clinically significant hypocalcemia after total thyroidectomy : a prospective study. Int J Current Res Rev. 2014;6(23):5-8.

18. Dralle H. [Thyroid gland surgery: risk factor surgeon]. Chirurg. 2012;83(3):280-281. doi:10.1007/s00104-012-2272-3

\section{Publish your work in this journal}

The International Journal of General Medicine is an international, peer-reviewed open-access journal that focuses on general and internal medicine, pathogenesis, epidemiology, diagnosis, monitoring and treatment protocols. The journal is characterized by the rapid reporting of reviews, original research and clinical studies across all disease areas. The manuscript management system is completely online and includes a very quick and fair peer-review system, which is all easy to use. Visit http://www.dovepress.com/ testimonials.php to read real quotes from published authors. 